\section{Discover metal-free RPD frames}

For a material that can enhance aesthetics, comfort, fit and function for removable partial dentures (RPD) take a look at the Ultaire AKP made by Solvay Dental 360.

This revolutionary new material has been specifically developed to meet the high performance needs of the dental field and has been custom designed to offer a strong but compliant alternative to metal cast frames.

Ultaire AKP is lightweight and biocompatible with bone-like properties that do not distort. It is elastic in tension so that it feels comfortable in the mouth but it also provides rigidity and stability needed for optimal fit and function.

Unlike metal-framed RPD, Ultaire $\mathrm{AKP}$ is taste neutral and it also eliminates any difficulties that patients may have regarding metal sensitivity. By supporting teeth on an Ultaire AKP RPD frame, point loading and uneven force can be avoided, as energy is dissipated across the entire framework.

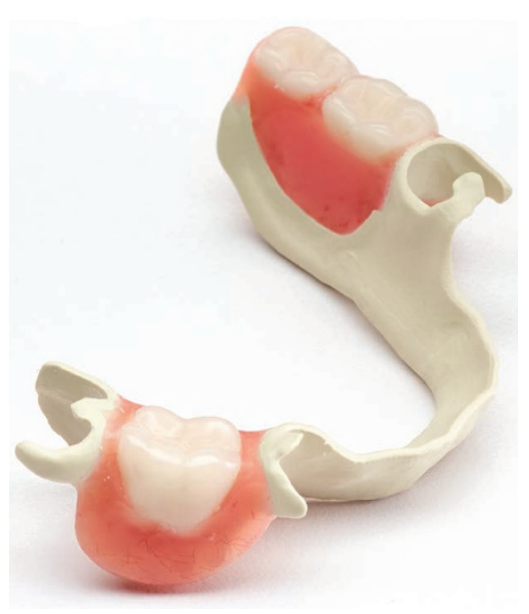

Ultaire AKP is great to work with - it is simple to adjust and articulate. It can be coloured and the Solvay Dental 360 specific clasp designs provide discreet retention with a 'snap in fit' and a natural look.

To please more patients, discover more about Ultaire AKP RPD frames now.

For more information about Solvay Dental 360, Ultaire AKP and Dentivera milling discs, visit www.solvaydental360.com.

\section{Catch a glimpse of the future}

Fast forward five, ten or even 20 years. What will the practice of the future look like? Dental Showcase 2017 is the perfect forum to take 'time out' and immerse in the certainties and possibilities of the future.

This year's Dental Showcase runs from 19-21 October in the NEC Birmingham. As always, this year it will host thousands of professionals as they discover the very latest dental innovations across an unrivalled selection of exhibitor stands. All attendees will enjoy personalised product demonstrations, have the opportunity to try out products, talk one-to-one with suppliers and be able to snap up unbeatable deals.

For the first time this year Showcase will unveil the Dental Practice of the Future. Equipped with reception area, a patient information zone and a fully working surgery, and fitted out with the very latest equipment and technology, it will give

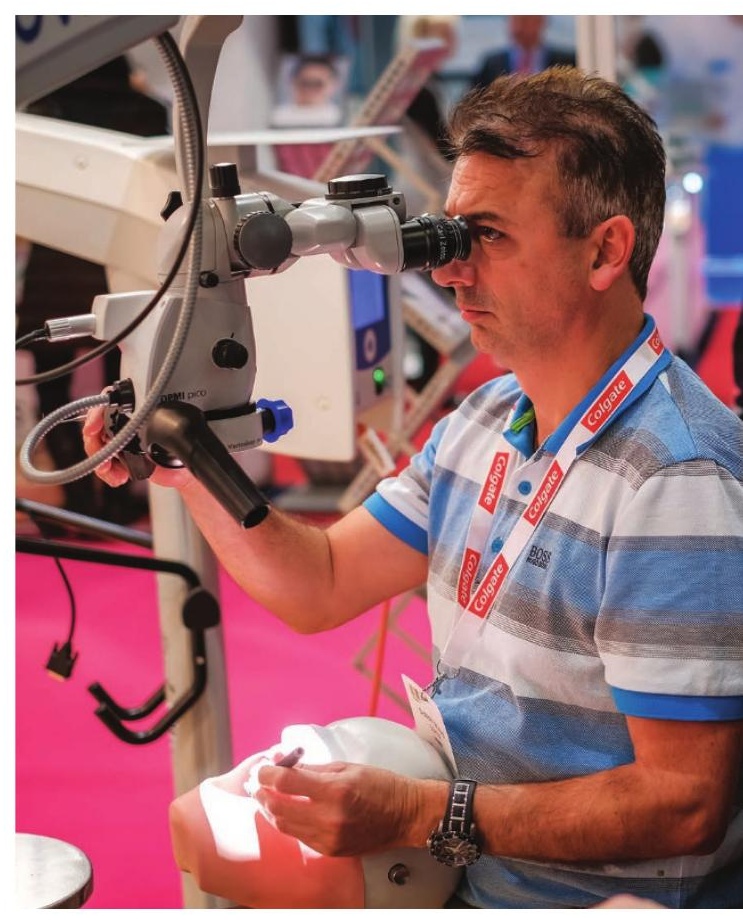
the whole practice team a window into the future. With a series of interactive demonstrations of the latest products and solutions, practice teams will be able to see first-hand how their working environment might evolve. The futuristic surgery will have seating, so that delegates can relax during keynote speeches and demonstrations and, while not in use, delegates will be free to look at the finer details and head to the stands to find out more about their favourites.

Combined with live demonstrations, interactive lectures, walk-arounds and panel discussions throughout the show floor, all practice members will be able to expand their knowledge of a vast range of subjects including medical emergencies, ergonomics, innovations in X-rays, 3D printing, purchasing, IRMER and much more.

Don't miss one of the most important dates in the dental calendar; give yourself the opportunity to experience it all first hand and register your place for this year's event at http://www. dentalshowcase.com/register.

\section{The finest in pin technology}

Filpin is engineered to provide the most advanced dentine retention pin design and performance made from pure (99.8\%) medical grade titanium for complete biocompatibility and compatibility with all dental materials.

Designed to be fast and easy to use, even in difficult places, Filpin will neither discolour nor corrode and can be bent as required with total confidence. Filpin shanks are autoclavable and may be reloaded many times with Filpin Spare Pin Inserts.

Filpin Pin Bender protects against damage to the pin and tooth structure. It is engineered for precision and accuracy with superior construction materials.
Filpost aids in the retention of restorations by root post and core build up.

Filpost preserves more healthy tooth structure and is stronger in use due to its unique passive 'interlocking' system. Filpost can be easily customised to suit the canal without risk of fracture, to tooth or restoration, enabling easy insertion of multiple posts into converging canals. No drilling is required during placement due to the special 'Universal Groover' that minimises dentine removal to create the unique passive interlock that strengthens retention and avoids the risk of perforation. Made of $99.8 \%$ pure titanium, filpost is design engineered to be easier, faster and safer to use. Available from your dental dealer. 